PROCEEDINGS OF THE

AMERICAN MATHEMATICAL SOCIETY

Volume 131, Number 9, Pages 2741-2751

S 0002-9939(03)07110-7

Article electronically published on April 23, 2003

\title{
CERTAIN EXTREMAL PROBLEMS FOR POLYNOMIALS
}

\author{
D. P. DRYANOV, M. A. QAZI, AND Q. I. RAHMAN
}

(Communicated by David Preiss)

\begin{abstract}
Extensions of two classical results about polynomials, one due to W. Markov and the other due to Duffin and Schaeffer, are obtained in this paper. An interesting result of S. Bernstein, which went unnoticed until it was rediscovered by P. Erdős, 34 years later, is also generalized. Our results are especially amenable to numerical calculations, and may, therefore, be of some practical importance.
\end{abstract}

\section{INTRODUCTION}

We shall show how a simple result, contained in Theorem 1 below, leads to some striking conclusions giving extensions and generalizations of the following two wellknown results. The first one is a classical result of Wladimir Markoff $([8, \S 17, \S 23]$; see also [6] and [9, p. 56]), and the second one is a famous result of Duffin and Schaeffer [4].

Theorem A. As usual, let

$$
T_{m}(x):=\frac{m}{2} \sum_{\mu=0}^{\lfloor m / 2\rfloor}(-1)^{\mu} \frac{(m-\mu-1) !}{\mu !(m-2 \mu) !}(2 x)^{m-2 \mu}=\cos (m \arccos x)
$$

be the Chebyshev polynomial of the first kind of degree $m$. In addition, let $f(x):=$ $\sum_{\nu=0}^{n} a_{\nu} x^{\nu}$ be a polynomial of degree at most $n$ such that $|f(x)| \leq 1$ for $-1 \leq x \leq$ 1. Then, $\left|a_{n-2 \mu}\right|$ is bounded above by the modulus of the corresponding coefficient of $T_{n}$ for $\mu=0, \ldots,\lfloor n / 2\rfloor$, and $\left|a_{n-1-2 \mu}\right|$ is bounded above by the modulus of the corresponding coefficient of $T_{n-1}$ for $\mu=0, \ldots,\lfloor(n-1) / 2\rfloor$.

Theorem B. Let $T_{n}$ be the Chebyshev polynomial of the first kind of degree $n$. In addition, let $f$ be a polynomial of degree at most $n$ such that $|f(x)| \leq 1$ at the zeros of the polynomial $\left(1-x^{2}\right) T_{n}^{\prime}(x)$. Then, for $1 \leq k \leq n$, we have

$$
\left|f^{(k)}(x+\mathrm{i} y)\right| \leq\left|T_{n}^{(k)}(1+\mathrm{i} y)\right| \quad(-1 \leq x \leq 1, y \in \mathbb{R}) .
$$

Received by the editors January 29, 2002.

2000 Mathematics Subject Classification. Primary 26C05, 26D05, 26D10.

Key words and phrases. Polynomials, inequalities, coefficient estimates, growth. 
We start with the following simple observation.

Lemma 1. Let $t_{0}<\cdots<t_{N}$, and define $P(x)=\prod_{\nu=0}^{N}\left(x-t_{\nu}\right)$. Then, for any polynomial $f$ of degree at most $N$ and any $\xi$ such that $P^{\prime}(\xi)=0$, we have

$$
f^{\prime}(\xi)=-P(\xi) \sum_{\nu=0}^{N} \frac{f\left(t_{\nu}\right)}{P^{\prime}\left(t_{\nu}\right)} \frac{1}{\left(\xi-t_{\nu}\right)^{2}} .
$$

Proof. By the Lagrange interpolation formula

$$
f(x) \equiv P(x) \sum_{\nu=0}^{N} \frac{f\left(t_{\nu}\right)}{P^{\prime}\left(t_{\nu}\right)} \frac{1}{x-t_{\nu}} .
$$

Differentiating the two sides of this identity with respect to $x$ and then replacing $x$ by $\xi$, we obtain

$$
f^{\prime}(\xi)=-P(\xi) \sum_{\nu=0}^{N} \frac{f\left(t_{\nu}\right)}{P^{\prime}\left(t_{\nu}\right)} \frac{1}{\left(\xi-t_{\nu}\right)^{2}}+P^{\prime}(\xi) \sum_{\nu=0}^{N} \frac{f\left(t_{\nu}\right)}{P^{\prime}\left(t_{\nu}\right)} \frac{1}{\xi-t_{\nu}},
$$

which is simply $(1)$, since $P^{\prime}(\xi)=0$.

With this, we are ready to prove the following result.

Theorem 1. Let $t_{0}<\cdots<t_{N}$ be an increasing sequence of $N+1$ real numbers, and let $y_{0}, \ldots, y_{N}$ be a set of $N+1$ non-negative numbers, not all zero. Let $\xi_{1}<\cdots<\xi_{N}$ be the critical points of $P(x):=\prod_{\nu=0}^{N}\left(x-t_{\nu}\right)$, and denote by $\pi_{N}$ the unique polynomial of degree at most $N$, which has the property that $\pi_{N}\left(t_{\nu}\right)=$ $(-1)^{N-\nu} y_{\nu}$ for $0 \leq \nu \leq N$. Furthermore, let $f$ be any polynomial of degree at most $N$ such that $\left|f\left(t_{\nu}\right)\right| \leq y_{\nu}$ for $0 \leq \nu \leq N$. Then

$$
\left|f^{\prime}\left(\xi_{\mu}\right)\right|<\left|\pi_{N}^{\prime}\left(\xi_{\mu}\right)\right|=(-1)^{N-\mu} \pi_{N}^{\prime}\left(\xi_{\mu}\right) \quad(1 \leq \mu \leq N),
$$

unless $f(x):=\mathrm{e}^{\mathrm{i} \gamma} \pi_{N}(x)$ for some real $\gamma$.

Proof. Note that

$$
P\left(\xi_{\mu}\right)=(-1)^{N-\mu+1}\left|P\left(\xi_{\mu}\right)\right| \quad(1 \leq \mu \leq N),
$$

and that $P^{\prime}\left(t_{\nu}\right)=(-1)^{N-\nu}\left|P^{\prime}\left(t_{\nu}\right)\right|$ for $0 \leq \nu \leq N$. Hence, (1) implies that

$$
f^{\prime}\left(\xi_{\mu}\right)=\left|P\left(\xi_{\mu}\right)\right| \sum_{\nu=0}^{N}(-1)^{\nu-\mu} \frac{f\left(t_{\nu}\right)}{\left|P^{\prime}\left(t_{\nu}\right)\right|} \frac{1}{\left(\xi_{\mu}-t_{\nu}\right)^{2}} \quad(1 \leq \mu \leq N) .
$$

Since $\pi_{N}\left(t_{\nu}\right)=(-1)^{N-\nu} y_{\nu}$ for $0 \leq \nu \leq N$, we see, in particular, that

$$
\pi_{N}^{\prime}\left(\xi_{\mu}\right)=(-1)^{N-\mu}\left|P\left(\xi_{\mu}\right)\right| \sum_{\nu=0}^{N} \frac{y_{\nu}}{\left|P^{\prime}\left(t_{\nu}\right)\right|} \frac{1}{\left(\xi_{\mu}-t_{\nu}\right)^{2}} \quad(1 \leq \mu \leq N) .
$$

From (3) and (4), it readily follows that for any $\xi_{\mu}$,

$$
\left|f^{\prime}\left(\xi_{\mu}\right)\right|<\left|\pi_{N}^{\prime}\left(\xi_{\mu}\right)\right| \quad(1 \leq \mu \leq N),
$$

unless $f(x):=\mathrm{e}^{\mathrm{i} \gamma} \pi_{N}(x)$ for some real $\gamma$. 


\section{Applications}

Theorems $2 a$ and $2 b$ are two parts of the same theorem. They are stated separately for the sake of clarity. Together, they constitute a multifaceted generalization of Theorem A. Theorem 3 also says a great deal more than Theorem A does. They all hold under much weaker assumptions, except Theorem 3 does not apply to polynomials with non-real coefficients.

Theorem 2a. Let $t_{0}<\cdots<t_{2 m}$ be a sequence of $2 m+1$ real numbers, such that $t_{\mu}=-t_{2 m-\mu}$ for $0 \leq \mu \leq m$. In addition, let $y_{0}, \ldots, y_{2 m}$ be a set of $2 m+1$ nonnegative numbers, not all zero, and subject to the requirement that $y_{\mu}=y_{2 m-\mu}$ for $0 \leq \mu \leq m$. Furthermore, let $F(x):=\sum_{\mu=0}^{2 m} A_{2 \mu} x^{2 \mu}$ be the unique (even) polynomial of degree $2 m$ such that $F\left(t_{\mu}\right)=(-1)^{2 m-\mu} y_{\mu}$ for $0 \leq \mu \leq 2 m$. Then, for any polynomial $p(x):=\sum_{\nu=0}^{2 m+1} a_{\nu} x^{\nu}$ of degree at most $2 m+1$ such that $\left|p\left(t_{\mu}\right)\right| \leq y_{\mu}$ for $0 \leq \mu \leq 2 m$, we have

$$
\left|a_{2 \mu}\right|=\frac{1}{(2 \mu) !}\left|p^{(2 \mu)}(0)\right|<\frac{1}{(2 \mu) !}\left|F^{(2 \mu)}(0)\right|=\left|A_{2 \mu}\right| \quad(1 \leq \mu \leq m),
$$

unless $p(x)+p(-x) \equiv 2 \mathrm{e}^{\mathrm{i} \gamma} F(x)$ for some real $\gamma$.

Proof. Clearly, $t_{m}=0$. Note that $f(x):=(p(x)+p(-x)) / 2=\sum_{\mu=0}^{m} a_{2 \mu} x^{2 \mu}$ is an even polynomial of degree not exceeding $2 m$ such that

$$
\left|f\left(t_{\mu}\right)\right| \leq y_{\mu} \quad(0 \leq \mu \leq 2 m) .
$$

Let $t_{0}^{(1)}, \ldots, t_{2 m-1}^{(1)}$ be the critical points of $P_{n, 0}(x):=\prod_{\mu=0}^{2 m}\left(x-t_{\mu}\right)$, where $t_{0}^{(1)}<\cdots<t_{2 m-1}^{(1)}$. Then, Theorem 1 applied with $N=2 m$ shows that

$$
\left|f^{\prime}\left(t_{\mu}^{(1)}\right)\right|<\left|F^{\prime}\left(t_{\mu}^{(1)}\right)\right| \quad(0 \leq \mu \leq 2 m-1),
$$

unless $f(x):=\mathrm{e}^{\mathrm{i} \gamma} F(x)$ for some real $\gamma$. Now, set $P_{n, 1}(x):=\prod_{\mu=0}^{2 m-1}\left(x-t_{\mu}^{(1)}\right)$ and denote its critical points by $t_{0}^{(2)}<\cdots<t_{2 m-2}^{(2)}$. It may be noted that $t_{m-1}^{(2)}=0$. In view of (7), Theorem 1 may be applied to conclude that if $f(x) \not \equiv \mathrm{e}^{\mathrm{i} \gamma} F(x)$ for all real $\gamma$, then

$$
\left|f^{\prime \prime}\left(t_{\mu}^{(2)}\right)\right|<\left|F^{\prime \prime}\left(t_{\mu}^{(2)}\right)\right| \quad(0 \leq \mu \leq 2 m-2) ;
$$

in particular,

$$
\left|f^{\prime \prime}(0)\right|<\left|F^{\prime \prime}(0)\right| \text {. }
$$

Comparing (8) with (6) and noting that $y_{\mu}=\left|F\left(x_{\mu}\right)\right|$ for $0 \leq \mu \leq 2 m$, we see that if $|f(x)| \leq|F(x)|$ at the zeros of $P_{n, 0}$, then $\left|f^{\prime \prime}(x)\right|<\left|F^{\prime \prime}(x)\right|$ at the zeros of $P_{n, 0}^{\prime \prime}$, unless $f(x) \equiv \mathrm{e}^{\mathrm{i} \gamma} F(x)$ for some real $\gamma$. Repeated use of the preceding argument shows that if $f(x) \not \equiv \mathrm{e}^{\mathrm{i} \gamma} F(x)$ for all real $\gamma$, then, at each of the $2(m-\mu)+1$ zeros of $P_{n, 0}^{(2 \mu)}$, we have

$$
\left|f^{(2 \mu)}(x)\right|<\left|F^{(2 \mu)}(x)\right| \quad(1 \leq \mu \leq m) ;
$$

in particular,

$$
\left|p^{(2 \mu)}(0)\right|=\left|f^{(2 \mu)}(0)\right|<\left|F^{(2 \mu)}(0)\right| \quad(1 \leq \mu \leq m) .
$$


Theorem 2b. Let $\tau_{0}<\cdots<\tau_{2 m-1}$ be $2 m$ real numbers, and let $y_{0}, \ldots, y_{2 m-1}$ be $2 m$ non-negative numbers, not all zero, such that $\tau_{\mu}=-\tau_{2 m-1-\mu}$ and $y_{\mu}=$ $y_{2 m-1-\mu}$ for $0 \leq \mu \leq m-1$. In addition, let $G(x):=\sum_{\mu=0}^{m-1} B_{2 \mu+1} x^{2 \mu+1}$ be the unique (odd) polynomial of degree $2 m-1$ such that $G\left(\tau_{\mu}\right)=(-1)^{2 m-\mu} y_{\mu}$ for $0 \leq \mu \leq 2 m-1$. Furthermore, let $p(x):=\sum_{\nu=0}^{2 m} a_{\nu} x^{\nu}$ be a polynomial of degree at most $2 m$ such that $\left|p\left(\tau_{\mu}\right)\right| \leq y_{\mu}$ for $0 \leq \mu \leq 2 m-1$. Then, for $0 \leq \mu \leq m-1$, we have

$$
\left|a_{2 \mu+1}\right|=\frac{1}{(2 \mu+1) !}\left|p^{(2 \mu+1)}(0)\right|<\frac{1}{(2 \mu+1) !}\left|G^{(2 \mu+1)}(0)\right|=\left|B_{2 \mu+1}\right|,
$$

unless $p(x)-p(-x) \equiv 2 \mathrm{e}^{\mathrm{i} \gamma} G(x)$ for some real $\gamma$.

Proof. Note that $g(x):=(p(x)-p(-x)) / 2=\sum_{\mu=0}^{m-1} a_{2 \mu+1} x^{2 \mu+1}$ is an odd polynomial of degree at most $2 m-1$ such that

$$
\left|g\left(\tau_{\mu}\right)\right| \leq y_{\mu} \quad(0 \leq \mu \leq 2 m-1) .
$$

Let $\tau_{0}^{(1)}, \ldots, \tau_{2 m-2}^{(1)}$ be the critical points of $Q_{n, 0}(x):=\prod_{\mu=0}^{2 m-1}\left(x-\tau_{\mu}\right)$, where $\tau_{0}^{(1)}<\cdots<\tau_{m-1}^{(1)}=0<\cdots<\tau_{2 m-2}^{(1)}$. Then, Theorem 1 may be applied with $N=2 m-1$ to conclude that if $g(x) \not \equiv \mathrm{e}^{\mathrm{i} \gamma} G(x)$ for all real $\gamma$, then

$$
\left|g^{\prime}\left(\tau_{\mu}^{(1)}\right)\right|<\left|G^{\prime}\left(\tau_{\mu}^{(1)}\right)\right| \quad(0 \leq \mu \leq 2 m-2)
$$

in particular,

$$
\left|g^{\prime}(0)\right|<\left|G^{\prime}(0)\right| \text {. }
$$

Now, let us compare (13) with (8). Like $f^{\prime \prime}$ and $F^{\prime \prime}$, the polynomials $g^{\prime}$ and $G^{\prime}$ are of degree at most $2 m-2$. Just as $\left|f^{\prime \prime}(x)\right|<\left|F^{\prime \prime}(x)\right|$ at the critical points of a polynomial of degree $2 m$, namely $P_{n, 1}$, the inequality $\left|g^{\prime}(x)\right|<\left|G^{\prime}(x)\right|$ holds at the critical points of a polynomial of degree $2 m$, namely $Q_{n, 0}$. Like the critical points of $P_{n, 1}$, those of $Q_{n, 0}$ are real and lie symmetrically with respect to the origin. Hence, in the same way as (8) leads to (5), inequality (13) implies (12).

Remark 1. Theorem A is contained in Theorems $2 a$ and $2 b$ put together. To see this, let $f(x):=\sum_{\nu=0}^{n} a_{\nu} x^{\nu}$ be as in Theorem A. Depending on whether $n$ is even or odd, we may apply Theorem $2 a$ or Theorem $2 b$, respectively, to the polynomial $p(x):=\sum_{\nu=0}^{n+1} a_{\nu} x^{\nu}$, where $a_{n+1}=0$, to obtain the first part of Theorem A. In order to obtain the second part, we may write $f(x)$ as $p(x):=\sum_{\nu=0}^{(n-1)+1} a_{\nu} x^{\nu}$, and then use Theorem $2 a$ or Theorem $2 b$, depending on the parity of $n-1$.

Remark 2. By a standard argument, the polynomial $p$ in Theorems $2 a$ and $2 b$ may be allowed to have non-real coefficients. The following result seems to be more interesting, if we were to restrict ourselves to real polynomials.

Theorem 3. Let $t_{0}<\cdots<t_{n}$ be $n+1$ real numbers, and let $y_{0}, \ldots, y_{n}$ be a sequence of $n+1$ non-negative numbers, where we suppose that $t_{\nu}=-t_{n-\nu}$ and $y_{\nu}=y_{n-\nu}$ for $\nu=0, \ldots, n$, and that $\sum_{\nu=0}^{n} y_{\nu}>0$. In addition, let $F(x):=\sum_{\mu=0}^{\lfloor n / 2\rfloor} A_{n-2 \mu} x^{n-2 \mu}$ be the unique polynomial of degree $n$ such that $F\left(t_{\nu}\right)=(-1)^{n-\nu} y_{\nu}$ for $\nu=0, \ldots, n$. Furthermore, let $p(x):=\sum_{\nu=0}^{n} a_{\nu} x^{\nu}$ be a real polynomial of degree at most $n$, whose modulus does not exceed that of $F$ at the points $t_{0}, \ldots, t_{n}$, that is,

$$
\left|p\left(t_{\nu}\right)\right| \leq y_{\nu}=\left|F\left(t_{\nu}\right)\right| \quad(0 \leq \nu \leq n)
$$


Then,

$$
\left|a_{n-2 \mu}\right|+\left|a_{n-2 \mu-1}\right| \leq\left|A_{n-2 \mu}\right| \quad\left(\mu=0, \ldots,\left\lfloor\frac{n-1}{2}\right\rfloor\right) .
$$

Proof. First, let $n$ be even and write $n=2 m$. Note that both

$$
f_{1}(x):=\frac{1}{2}\{(1+x) p(x)+(1-x) p(-x)\}=a_{0}+\sum_{\mu=1}^{m}\left(a_{2 \mu}+a_{2 \mu-1}\right) x^{2 \mu}
$$

and

$$
f_{2}(x):=\frac{1}{2}\{(1-x) p(x)+(1+x) p(-x)\}=a_{0}+\sum_{\mu=1}^{m}\left(a_{2 \mu}-a_{2 \mu-1}\right) x^{2 \mu}
$$

satisfy the conditions of Theorem $2 a$, and so for $1 \leq \mu \leq m$, we have

$$
\left|a_{2 \mu}+a_{2 \mu-1}\right| \leq\left|A_{2 \mu}\right|,\left|a_{2 \mu}-a_{2 \mu-1}\right| \leq\left|A_{2 \mu}\right| .
$$

Since the coefficients $a_{0}, \ldots, a_{n}$ are all real,

$$
\left|a_{2 \mu}\right|+\left|a_{2 \mu-1}\right|=\max \left\{\left|a_{2 \mu}+a_{2 \mu-1}\right|,\left|a_{2 \mu}-a_{2 \mu-1}\right|\right\},
$$

and so (14) holds.

Next, let $n$ be odd and write $n=2 m-1$. In this case the functions

$$
f_{3}(x):=\frac{1}{2}\{(1+x) p(x)-(1-x) p(-x)\}=\sum_{\mu=1}^{m-1}\left(a_{2 \mu+1}+a_{2 \mu}\right) x^{2 \mu+1}
$$

and

$$
f_{4}(x):=\frac{1}{2}\{(1-x) p(x)-(1+x) p(-x)\}=\sum_{\mu=1}^{m}\left(a_{2 \mu+1}-a_{2 \mu}\right) x^{2 \mu+1}
$$

satisfy the conditions of Theorem $2 a$, and so for $0 \leq \mu \leq m-1$, we have

$$
\left|a_{2 \mu+1}+a_{2 \mu}\right| \leq\left|A_{2 \mu+1}\right|,\left|a_{2 \mu+1}-a_{2 \mu}\right| \leq\left|A_{2 \mu+1}\right| .
$$

Since the coefficients $a_{0}, \ldots, a_{n}$ are all real,

$$
\left|a_{2 \mu+1}\right|+\left|a_{2 \mu}\right|=\max \left\{\left|a_{2 \mu+1}+a_{2 \mu}\right|,\left|a_{2 \mu+1}-a_{2 \mu}\right|\right\},
$$

and so, once again, (14) holds.

Remark 3. It is known [5, p. 1176] that if $p(z):=\sum_{k=0}^{n} a_{k} z^{k}$ has real coefficients and $|p(z)| \leq 1$ for $-1 \leq x \leq 1$, then $\sum_{k=0}^{n}\left|a_{k}\right|$ is maximal for $p(z):= \pm T_{n}(z)$. According to an oral communication to Erdős (see [5] p. 1176]), G. Szegö could even prove that $\left|a_{2 k}\right|+\left|a_{2 k+1}\right|$ is maximal for $p(z):= \pm T_{n}(z)$. It may be noted that this result of Szégö is a very special case of Theorem 3. It also fully covers Theorem A.

The following result is due to Bernstein [1. It remained unknown until it was rediscovered by Erdős [5, Theorem 7].

Theorem C. Let $f$ be a polynomial of degree $n$ with real coefficients, such that $|f(x)| \leq 1$ for $-1 \leq x \leq 1$. Then

$$
|f(z)| \leq\left|T_{n}(z)\right| \quad(|z| \geq 1) .
$$

Equality holds only for $f(z)= \pm T_{n}(z)$. 
As an extension of Theorem $\mathrm{C}$ we prove the following result.

Theorem 4. Let $t_{0}<\cdots<t_{N}$ be an arbitrary set of $N+1$ real numbers, and let $y_{0}, \ldots, y_{N}$ be any set of $N+1$ non-negative numbers, not all zero. Denote by $\pi_{N}$ the unique polynomial of degree at most $N$ such that $\pi_{N}\left(t_{\nu}\right)=(-1)^{N-\nu} y_{\nu}$ for $0 \leq \nu \leq N$. Furthermore, let $f$ be a real polynomial of degree at most $N$ such that $\left|f\left(t_{\nu}\right)\right| \leq y_{\nu}$ for $0 \leq \nu \leq N$. Then,

$$
|f(z)|<\left|\pi_{N}(z)\right| \quad\left(\left|z-\frac{t_{0}+t_{N}}{2}\right| \geq \frac{t_{N}-t_{0}}{2}, z \notin\left\{t_{0}, t_{N}\right\}\right),
$$

unless $f(z) \equiv \pm \pi_{N}(z)$.

Proof. Without loss of generality we may suppose that $t_{0}=-1$ and $t_{N}=1$. Now, let $z \neq \pm 1$ be any point such that $|z| \geq 1$. Introducing the fundamental functions

$$
\ell_{k}(z):=\prod_{\substack{j=0 \\ j \neq k}}^{N} \frac{z-t_{j}}{t_{k}-t_{j}} \quad(k=0, \ldots, N)
$$

we may use the Lagrange interpolation formula to write

$$
f(z)=\sum_{k=0}^{N} f\left(t_{k}\right) \ell_{k}(z)=\sum_{k=0}^{N}(-1)^{k} f\left(t_{k}\right) \cdot(-1)^{k} \ell_{k}(z) .
$$

Note that for $0 \leq \mu \neq \nu \leq N$, we have

$$
\frac{(-1)^{\mu} \ell_{\mu}(z)}{(-1)^{\nu} \ell_{\nu}(z)}=\frac{(-1)^{N-\mu} \ell_{\mu}(z)}{(-1)^{N-\nu} \ell_{\nu}(z)}=c_{\mu, \nu} \frac{t_{\nu}-z}{t_{\mu}-z}
$$

where $c_{\mu, \nu}>0$. Since the angle subtended by $\left[t_{0}, t_{N}\right]$ at the point $z$ does not exceed $\pi / 2$, the vectors $t_{\nu}-z, t_{\mu}-z$, which are non-zero, lie in a sector of opening at most $\pi / 2$ for $0 \leq \mu \neq \nu \leq N$. So, from (17), it follows that all the vectors $(-1)^{0} \ell_{0}(z), \ldots,(-1)^{N} \ell_{N}(z)$ must also lie in some sector of opening not exceeding $\pi / 2$. Hence, for any $z \notin\left\{t_{0}, t_{N}\right\}$ outside the open disk with diameter $\left[t_{0}, t_{N}\right]$, there exists a real number $\beta$ such that

$$
u_{k}:=\Re\left((-1)^{k} \ell_{k}(z) \mathrm{e}^{-\mathrm{i} \beta}\right) \geq 0 \quad \text { and } \quad v_{k}:=\Im\left((-1)^{k} \ell_{k}(z) \mathrm{e}^{-\mathrm{i} \beta}\right) \leq 0
$$

for $k=0, \ldots, N$. Hence,

$$
\begin{aligned}
|f(z)|^{2} & =\left|f(z) \mathrm{e}^{-\mathrm{i} \beta}\right|^{2}=\left|\sum_{k=0}^{N}(-1)^{k} f\left(t_{k}\right)\left(u_{k}+\mathrm{i} v_{k}\right)\right|^{2} \\
& =\left(\sum_{k=0}^{N}(-1)^{k} f\left(t_{k}\right) u_{k}\right)^{2}+\left(\sum_{k=0}^{N}(-1)^{k} f\left(t_{k}\right) v_{k}\right)^{2} \\
& \leq\left(\sum_{k=0}^{N} y_{k} u_{k}\right)^{2}+\left(\sum_{k=0}^{N} y_{k} v_{k}\right)^{2}=\left|\sum_{k=0}^{N}(-1)^{k} y_{k} \ell_{k}(z)\right|^{2}=\left|\pi_{N}(z)\right|
\end{aligned}
$$

where the inequality is strict unless $(-1)^{0} f\left(t_{0}\right) y_{0}, \ldots,(-1)^{N} f\left(t_{N}\right) y_{N}$ are all of the same sign. This is not possible unless $f(z) \equiv \pm \pi_{N}(z)$. 
Using Theorem 1 in conjunction with Theorem 4, we deduce the following result.

Theorem 5. Let $t_{0}<\cdots<t_{N}$ be an arbitrary set of $N+1$ real numbers, and let $P_{N, 0}(z):=\prod_{\nu=0}^{N}\left(z-t_{\nu}\right)$. In addition, let $y_{0}, \ldots, y_{N}$ be any set of $N+1$ non-negative numbers, not all zero, and denote by $\pi_{N}$ the unique polynomial of degree at most $N$ such that $\pi_{N}\left(t_{\nu}\right)=(-1)^{N-\nu} y_{\nu}$ for $0 \leq \nu \leq N$. Finally, let $t_{0, k}<\cdots<t_{N-k, k}$ be the zeros of $P_{N, 0}^{(k)}$. Then for any real polynomial $f$ of degree at most $N$ such that $\left|f\left(t_{\nu}\right)\right| \leq y_{\nu}$ for $0 \leq \nu \leq N$, the inequality

$$
\left|f^{(k)}(z)\right|<\left|\pi_{N}^{(k)}(z)\right| \quad(k=1, \ldots, N)
$$

holds for all $z \notin\left\{t_{0, k}, t_{N-k, k}\right\}$ such that

$$
\left|z-\frac{t_{0, k}+t_{N-k, k}}{2}\right| \geq \frac{t_{N-k, k}-t_{0, k}}{2}
$$

unless $f(z) \equiv \pm \pi_{N}(z)$.

Proof. Let $f \neq \pm \pi_{N}$. By Theorem 1, $\left|f^{\prime}\left(t_{\nu, 1}\right)\right|<\left|\pi_{N}^{\prime}\left(t_{\nu, 1}\right)\right|$ for $0 \leq \nu \leq N-1$, which in turn implies that $\left|f^{\prime \prime}\left(t_{\nu, 2}\right)\right|<\left|\pi_{N}^{\prime \prime}\left(t_{\nu, 2}\right)\right|$ for $0 \leq \nu \leq N-2$. In fact, for $1 \leq k \leq N$, we have

$$
\left|f^{(k)}\left(t_{\nu, k}\right)\right|<\left|\pi_{N}^{(k)}\left(t_{\nu, k}\right)\right| \quad(0 \leq \nu \leq N-k) .
$$

Hence, by Theorem 4,

$$
\left|f^{\prime}(z)\right|<\left|\pi_{N}^{\prime}(z)\right|
$$

for all $z \notin\left\{t_{0,1}, t_{N-1,1}\right\}$ such that

$$
\left|z-\frac{t_{0,1}+t_{N-1,1}}{2}\right| \geq \frac{t_{N-1,1}-t_{0,1}}{2} .
$$

More generally, if $1 \leq k \leq N$, then

$$
\left|f^{(k)}(z)\right|<\left|\pi_{N}^{(k)}(z)\right| \quad(k=1, \ldots, N)
$$

for any $z$ outside the open disk

$$
\left|z-\frac{t_{0, k}+t_{N-k, k}}{2}\right|<\frac{t_{N-k, k}-t_{0, k}}{2}
$$

other than $t_{0, k}$ and $t_{N-k, k}$.

Remark 4. Theorem 5 says in particular that if $f$ is as in Theorem B and $t_{n-k, k}$ is the largest zero of the polynomial

$$
\frac{\mathrm{d}^{k}}{\mathrm{~d} x^{k}}\left\{\left(1-x^{2}\right) T_{n}^{\prime}(x)\right\}, \quad \text { that is, of } \quad x T_{n}^{(k)}(x)+\left(n^{2}+k-1\right) T_{n}^{(k-1)}(x),
$$

then $\left|f^{(k)}(z)\right| \leq\left|T_{n}^{(k)}(z)\right|$ for $|z| \geq t_{n-k, k}$.

An addendum to Theorem 4. It is clear that in Theorem 4 we cannot expect $|f(z)|$ to be less than $\left|\pi_{N}(z)\right|$, at least at those points of the open disk $\mathfrak{D}$ of radius $\left(t_{N}-t_{0}\right) / 2$ centred at $\left(t_{0}+t_{N}\right) / 2$, where $\pi_{N}(z)$ vanishes. It is an interesting problem to find the sharp estimate for $|f(z)|$ at any point of $\mathfrak{D}$ if $f$ satisfies the conditions of Theorem 4. Instead of dealing with the problem in its full generality, we shall give just one result, which shows how the extremals may change from one point to the other. 
Theorem 6. Let $t_{0}<\cdots<t_{N}$ be an arbitrary set of $N+1$ real numbers, and let $y_{0}, \ldots, y_{N}$ be any set of $N+1$ non-negative numbers, not all zero. For any $m$ in $\{0,1, \ldots, N-1\}$, let $\pi_{N, m}$ be the unique polynomial of degree at most $N$ such that $\pi_{N, m}\left(t_{\nu}\right)=(-1)^{\nu} y_{\nu}$ for $0 \leq \nu \leq m$ and $\pi_{N, m}\left(t_{\nu}\right)=(-1)^{\nu-1} y_{\nu}$ for $m+1 \leq \nu \leq N$. Furthermore, let $f$ be a real polynomial of degree at most $N$ such that $\left|f\left(t_{\nu}\right)\right| \leq y_{\nu}$ for $0 \leq \nu \leq N$. Then,

$$
|f(z)|<\left|\pi_{N, m}(z)\right| \quad\left(\left|z-\frac{t_{m}+t_{m+1}}{2}\right| \leq \frac{t_{m+1}-t_{m}}{2}, z \notin\left\{t_{m}, t_{m+1}\right\}\right),
$$

unless $f(z) \equiv \pm \pi_{N, m}(z)$.

Proof. Let us denote the points $t_{m}$ and $t_{m+1}$ by $A$ and $B$, respectively, and the closed disk with $A B$ as diameter by $\overline{\mathfrak{D}}_{m}$. We have to show that if $C$ is any point of $\overline{\mathfrak{D}}_{m}$, other than $A$ and $B$, then $|f(C)|<\left|\pi_{N, m}(C)\right|$, unless $f(z) \equiv \pm \pi_{N, m}(z)$. As before, let $P(z)=\prod_{\nu=0}^{N}\left(z-t_{\nu}\right)$. Then, by the Lagrange interpolation formula

$$
\begin{aligned}
f(z)=\sum_{\nu=0}^{N} f\left(t_{\nu}\right) \frac{P(z)}{P^{\prime}\left(t_{\nu}\right)\left(z-t_{\nu}\right)}=(-1)^{N-1} P(z)\left\{\sum_{\nu=0}^{m} \frac{(-1)^{\nu} f\left(t_{\nu}\right)}{\left|P^{\prime}\left(t_{\nu}\right)\right|}\left(-\frac{1}{z-t_{\nu}}\right)\right. \\
\left.+\sum_{\nu=m+1}^{N} \frac{(-1)^{\nu-1} f\left(t_{\nu}\right)}{\left|P^{\prime}\left(t_{\nu}\right)\right|} \frac{1}{z-t_{\nu}}\right\} .
\end{aligned}
$$

Let $C$ represent the number $z_{0}$, and first let $\Im z_{0}>0$. Extend the directed segment $\overrightarrow{B C}$, and take a point $L$ on the extended part. Denote by $S$ the open sector determined by the line segments $\overrightarrow{C A}$ and $\overrightarrow{C L}$, and note that the angle $\angle A C L$ is at most $90^{\circ}$. Let $\alpha, \beta$ and $\gamma$ represent the radian measures of the internal angles of the triangle $A B C$ at $A, B$ and $C$, respectively. It is easily seen that the vectors $-1 /\left(z_{0}-t_{\nu}\right)$, where $\nu=0, \ldots, m$, and the vectors $1 /\left(z_{0}-t_{\nu}\right)$, where $\nu=m+1, \ldots, N$, all lie in the sector $\left\{z=z_{0}+r \mathrm{e}^{\mathrm{i} \theta}: r \geq 0, \pi-\alpha \leq \theta \leq \pi+\beta\right\}$ whose opening is the same as that of $S$, that is, $\alpha+\beta=\pi-\gamma \leq \pi / 2$. Hence, $\left|f\left(z_{0}\right)\right|$ is maximized by $\pm f$, where $f$ is the unique polynomial $\pi_{N, m}$ of degree at most $N$ for which $(-1)^{\nu} f\left(t_{\nu}\right)=y_{\nu}$ for $\nu=0, \ldots, m$, and $(-1)^{\nu} f\left(t_{\nu}\right)=-y_{\nu}$ for $\nu=m+1, \ldots, N$. For any other polynomial, the value of $\left|f\left(z_{0}\right)\right|$ must be smaller. By symmetry, the same is true when $\Im z_{0}<0$. The argument easily extends to cover the case where $t_{m}<z_{0}<t_{m+1}$.

An integral inequality. If $f$ is a real polynomial of degree at most $n$ such that $|f(\cos (\nu \pi / n))| \leq 1$ for $\nu=0, \ldots, n$, then, clearly, $|f(z)|$ may be larger than $\left|T_{n}(z)\right|$ at certain points of the unit disk, for example at points which are close to the zeros of $T_{n}$. It therefore seems interesting that if $\mathfrak{E}_{R}$ denotes the ellipse whose foci lie at $1,-1$ and the sum of whose semi-axes is $R$, where $R \geq \sqrt{2+1 / 2^{2 n-3}}$, then the integral

$$
I_{R}=I_{R}(f):=\int_{\mathfrak{E}_{R}}|f(\zeta)|^{2} \frac{|\mathrm{d} \zeta|}{\left|\sqrt{\zeta^{2}-1}\right|}
$$

is maximized by $T_{n}$ if simply

$$
\mathfrak{M}_{1}(f):=\frac{1}{n}\left\{\frac{1}{2}|f(1)|^{2}+\sum_{\nu=1}^{n-1}\left|f\left(\cos \frac{\nu \pi}{n}\right)\right|^{2}+\frac{1}{2}|f(-1)|^{2}\right\} \leq 1=\mathfrak{M}_{1}\left(T_{n}\right)
$$


whether the coefficients of $f$ are all real or not. The following result contains the sharp estimate for $I_{R}$ for every $R>1$, under the assumption that (20) holds. Note that (20) is certainly satisfied if $|f(\cos (\nu \pi / n))| \leq 1$ for $\nu=0, \ldots, n$.

Theorem 7. Let $f$ be a polynomial of degree at most $n$ such that (20) holds, and let $R_{n}$ be the only root of the equation

$$
r^{4 n}-2 r^{4 n-2}-2 r^{2}+1=0
$$

in $(1, \infty)$. Furthermore, let $\mathfrak{E}_{R}$ denote the ellipse whose foci lie at $1,-1$ and the sum of whose semi-axes is $R$. Then

$$
I_{R}(f):=\int_{\mathfrak{E}_{R}}|f(\zeta)|^{2} \frac{|\mathrm{d} \zeta|}{\left|\sqrt{\zeta^{2}-1}\right|} \leq \begin{cases}\pi\left(R^{2 n}+R^{-2 n}\right) / 2 & \text { if } R \geq R_{n}, \\ \pi\left(R^{2 n-2}+R^{-(2 n-2)}\right) & \text { if } 1 \leq R \leq R_{n}\end{cases}
$$

Proof. If $\zeta=\left(z+z^{-1}\right) / 2$, then $f(\zeta)=F(z):=\sum_{\nu=-n}^{n} b_{\nu} z^{\nu}$, where $b_{-\nu}=b_{\nu}$ for $\nu=0, \pm 1, \ldots, \pm n$. Hence, for any $R>1$, we have

$$
\begin{aligned}
I_{R}=I_{R}(f) & =\int_{|z|=R}|F(z)|^{2}\left|\frac{1-z^{-2}}{2}\right| \frac{|d z|}{\left|\left(z-z^{-1}\right) / 2\right|}=\int_{-\pi}^{\pi}\left|F\left(R \mathrm{e}^{\mathrm{i} \theta}\right)\right|^{2} \mathrm{~d} \theta \\
& =2 \pi \sum_{\nu=-n}^{n}\left|b_{\nu}\right|^{2} R^{2 \nu}=2 \pi\left\{\left|b_{0}\right|^{2}+\sum_{\nu=1}^{n}\left(R^{2 \nu}+R^{-2 \nu}\right)\left|b_{\nu}\right|^{2}\right\} .
\end{aligned}
$$

Thus, we obtain a representation for $I_{R}$ in terms of $\left|b_{0}\right|^{2}, \ldots,\left|b_{n}\right|^{2}$. Next, we interpret (20) as a condition on these parameters. For this we first note that

$$
|f(\cos \theta)|^{2}=f(\cos \theta) \overline{f(\cos \theta)}=\left|b_{n}\right|^{2} \mathrm{e}^{2 \mathrm{i} n \theta}+\cdots+\sum_{\nu=-n}^{n}\left|b_{\nu}\right|^{2}+\cdots+\left|b_{n}\right|^{2} \mathrm{e}^{-2 \mathrm{in} n}
$$

Remarking that $\sum_{k=0}^{2 n-1}\left(\mathrm{e}^{2 k \pi \mathrm{i} / 2 n}\right)^{\nu}=0$ for $\nu= \pm 1, \ldots, \pm(2 n-1)$, and

$$
\sum_{k=0}^{2 n-1}\left(\mathrm{e}^{2 k \pi \mathrm{i} / 2 n}\right)^{2 n}=\sum_{k=0}^{2 n-1}\left(\mathrm{e}^{2 k \pi \mathrm{i} / 2 n}\right)^{0}=\sum_{k=0}^{2 n-1}\left(\mathrm{e}^{2 k \pi \mathrm{i} / 2 n}\right)^{-2 n}=2 n,
$$

we conclude that

$$
\sum_{k=0}^{2 n-1}\left|f\left(\cos \frac{k \pi}{n}\right)\right|^{2}=2 n\left|b_{n}\right|^{2}+2 n \sum_{\nu=-n}^{n}\left|b_{\nu}\right|^{2}+2 n\left|b_{n}\right|^{2}
$$

Hence,

$$
4\left|b_{n}\right|^{2}+2 \sum_{\nu=1}^{n-1}\left|b_{n-\nu}\right|^{2}+\left|b_{0}\right|^{2}=2\left|b_{n}\right|^{2}+\sum_{\nu=-n}^{n}\left|b_{\nu}\right|^{2}=\mathfrak{M}_{1}(f) \leq 1 .
$$

Writing $t_{\nu}=\left|b_{\nu}\right|^{2}$ for $\nu=0, \ldots, n$, our problem reduces to maximization of the objective function

$$
\Phi\left(t_{0}, \ldots, t_{n}\right)=2 \pi\left\{t_{0}+\sum_{\nu=1}^{n}\left(R^{2 \nu}+R^{-2 \nu}\right) t_{\nu}\right\}
$$

subject to the condition that $t_{\nu} \geq 0$ for $\nu=0, \ldots, n$, and that

$$
4 t_{n}+2 \sum_{\nu=1}^{n-1} t_{n-\nu}+t_{0}=\alpha \leq 1 .
$$


The Fundamental Theorem of Linear Programming [5] applies to this problem and readily shows that for any $R>1$, the quantity $\Phi\left(t_{0}, \ldots, t_{n}\right)$ cannot be larger than

$$
\max \left\{\pi \frac{R^{2 n}+R^{-2 n}}{2}, \pi\left(R^{2 n-2}+R^{-(2 n-2)}\right), \ldots, \pi\left(R^{2}+R^{-2}\right), 2 \pi\right\} .
$$

This gives us the desired result.

The upper bound for $I_{R}$, given in (22), is attained for $\mathrm{e}^{\mathrm{i} \gamma} T_{n}, \gamma \in \mathfrak{R}$, or for $\mathrm{e}^{\mathrm{i} \gamma} \sqrt{2} T_{n-1}, \gamma \in \mathfrak{R}$, according as $R \geq R_{n}$ or $R_{n} \geq R>1$, respectively.

Remark 5. Instead of using the properties of the $2 n$-th roots of unity as we have done in the above proof, we could have used the Lobatto-Chebyshev quadrature from the theory of numerical integration.

Another pointwise condition. Even if $|f(x)|$ was bounded by 1, not only at the extrema of $T_{n}$, but at all the points of the unit interval, the integral $I_{R}$ would not be maximized by $T_{n}$ for all $R>1$. In fact, the value of $I_{R}$, corresponding to the constant polynomial 1, is larger than $\pi\left(R^{2 n}+R^{-2 n}\right) / 2$ for $1<R \leq((2+\sqrt{3}))^{1 / 2 n}$. It therefore seems interesting to mention a pointwise condition under which the functional $I_{R}$ is maximized by $T_{n}$ for all $R>1$.

Theorem 8. Let $f$ be a polynomial of degree at most $n$ such that $|f(x)|$ is bounded by $\left|T_{n}(x)\right|$ at the points $x=\cos (2 k \pi /(2 n+1))$ for $k=0, \ldots, n$, or at least

$$
\mathfrak{M}_{2}(f):=\frac{1}{2 n+1}\left\{|f(1)|^{2}+2 \sum_{k=1}^{n}\left|f\left(\cos \frac{2 k \pi}{2 n+1}\right)\right|^{2}\right\} \leq \mathfrak{M}_{2}\left(T_{n}\right) .
$$

Then, for any $R>1$, the functional $I_{R}(f)$ of $(22)$ is maximized only by polynomials of the form $\mathrm{e}^{\mathrm{i} \gamma} T_{n}$, where $\gamma$ is real.

Proof. We recall that $I_{R}=2 \pi\left\{\left|b_{0}\right|^{2}+\sum_{\nu=1}^{n}\left(R^{2 \nu}+R^{-2 \nu}\right)\left|b_{\nu}\right|^{2}\right\}$, where $b_{0}, \ldots, b_{n}$ are as in the proof of Theorem 7. In order to display their dependence on $f$, let us write $b_{\nu}=b_{\nu}(f)$ for $\nu=0, \pm 1, \ldots, \pm n$. Note that $R^{2 \nu}+R^{-2 \nu}$ is an increasing function of $\nu$, and so, $I_{R}(f) \leq \pi\left(R^{2 n}+R^{-2 n}\right) \sum_{\nu=-n}^{n}\left|b_{\nu}(f)\right|^{2}$ for all $R>1$, where equality holds for any $R$ only if all the coefficients except $b_{n}$ and $b_{-n}$ are zero. Since $\left(z^{\nu}+z^{-\nu}\right) / 2=T_{\nu}\left(\left(z+z^{-1}\right) / 2\right)$, this would mean that $f\left(\left(z+z^{-1}\right) / 2\right)$ is equal to $2 b_{n} T_{n}\left(\left(z+z^{-1}\right) / 2\right)$ for any $z \neq 0$, but then $f$ must be the same as $2 b_{n} T_{n}$. Here, for an obvious reason, $2 b_{n}$ must be of the form $\mathrm{e}^{\mathrm{i} \gamma}$ for some real $\gamma$. Since $\sum_{k=0}^{n}\left(\mathrm{e}^{2 k \pi \mathrm{i} /(2 n+1)}\right)^{\nu}=0$ for $\nu= \pm 1, \ldots, \pm 2 n$, it follows from (23) in conjunction with (24) that $\sum_{\nu=-n}^{n}\left|b_{\nu}(f)\right|^{2}=\mathfrak{M}_{2}(f) \leq \mathfrak{M}_{2}\left(T_{n}\right)=\sum_{\nu=-n}^{n}\left|b_{\nu}\left(T_{n}\right)\right|^{2}$. Hence,

$$
I_{R}(f) \leq \pi\left(R^{2 n}+R^{-2 n}\right) \sum_{\nu=-n}^{n}\left|b_{\nu}\left(T_{n}\right)\right|^{2}=\pi \frac{R^{2 n}+R^{-2 n}}{2}=I_{R}\left(\mathrm{e}^{\mathrm{i} \gamma} T_{n}\right) .
$$

Remark 6. If we did not care to keep the proof self-contained, we could have used the Radau-Chebyshev rule, instead of a property of the $(2 n+1)$-th roots of unity.

Remark 7. Although we quote here only two of their papers, namely [2] and [3], similar results appear in several papers of B. D. Bojanov and his students. 


\section{REFERENCES}

[1] S.N. Bernstein, Sur une propriété des polynômes, Comm. Soc. Math. Kharkow Sér. 214 (1913), pp. 1-6.

[2] B.D. Bojanov, An inequality of Duffin Schaeffer type, East J. Approx. 1 (1995), pp. 37-46. MR 97g:41016

[3] B.D. Bojanov and G. Nikolov, Duffin and Schaeffer type inequality for ultraspherical polynomials, J. Approx. Theory 84 (1996), pp. 129-138. MR 96k:41013

[4] R.J. Duffin and A.C. Schaeffer, A refinement of an inequality of the brothers Markoff, Trans. Amer. Math. Soc. 50 (1941), 517-528. MR 3:235c

[5] P. Erdős, Some remarks on polynomials, Bull. Amer. Math. Soc. 53 (1947), 1169-1176. MR 9:281g

[6] O.D. Kellogg, On bounded polynomials in several variables, Math. Z. 27 (1928), 55-64.

[7] D.G. Luenberger, Linear and Nonlinear Programming, 2nd ed., Addison-Wesley Publishing Company, Reading, Massachusetts, 1984.

[8] W. Markoff, Über Polynome, die in einem gegebenen Intervalle möglichst wenig von Null abweichen, Math. Ann. 77 (1916), 213-258.

[9] I.P. Natanson, Constructive function theory, Vol. I (Uniform approximation), Frederick Ungar Publishing Co., New York, 1964. MR 33:4529a

Département de Mathématiques et de Statistique, Université de Montréal, Montréal, QuÉBec, Canada H3C 3J7

E-mail address: drynovd@dms.umontreal.ca

Department of Mathematics, Tuskegee University, Tuskegee, Alabama 36088

E-mail address: qazima@aol.com

Département de Mathématiques et de Statistique, Université de Montréal, Montréal, QuéBec, CANAdA H3C 3J7

E-mail address: rahmanqi@dms.umontreal.ca 